\title{
Differential Impacts of Soybean and Fish Oils on Hepatocyte Lipid Droplet Accumulation and Endoplasmic Reticulum Stress in Primary Rabbit Hepatocytes
}

\author{
Xueping Zhu, ${ }^{1}$ Zhihui Xiao, ${ }^{1}$ Yumin Xu, ${ }^{1}$ Xingli Zhao, ${ }^{1}$ Ping Cheng, ${ }^{1}$ Ningxun Cui, ${ }^{1}$ \\ Mingling Cui, ${ }^{1}{ }^{\text {Jie Li, }}{ }^{1}$ and Xiaoli $\mathrm{Zhu}^{2}$
}

${ }^{1}$ Department of Neonatology, Children's Hospital of Soochow University, Suzhou, Jiangsu 215003, China

${ }^{2}$ Department of Intervention, The First Affiliated Hospital of Soochow University, Suzhou 215006, China

Correspondence should be addressed to Xueping Zhu; zhuxueping4637@hotmail.com and Xiaoli Zhu; zhuxiaoli90@hotmail.com

Received 20 July 2015; Accepted 29 October 2015

Academic Editor: John N. Plevris

Copyright ( $) 2016$ Xueping Zhu et al. This is an open access article distributed under the Creative Commons Attribution License, which permits unrestricted use, distribution, and reproduction in any medium, provided the original work is properly cited.

Parenteral nutrition-associated liver disease (PNALD) is a severe ailment associated with long-term parenteral nutrition. Soybean oil-based lipid emulsions (SOLE) are thought to promote PNALD development, whereas fish oil-based lipid emulsions (FOLE) are thought to protect against PNALD. This study aimed to investigate the effects of SOLE and FOLE on primary rabbit hepatocytes. The results reveal that SOLE caused significant endoplasmic reticulum (ER) and mitochondrial damage, ultimately resulting in lipid droplets accumulation and ER stress. While these deleterious events induce hepatocyte injury, FOLE at high doses cause only minor ER and mitochondrial damage, which has no effect on hepatic function. SOLE also significantly upregulated glucose-regulated protein 94 mRNA and protein expression. These data indicate that SOLE, but not FOLE, damage the ER and mitochondria, resulting in lipid droplets accumulation and ER stress and, finally, hepatocyte injury. This likely contributes to the differential impacts of SOLE and FOLE on PNALD development and progression.

\section{Introduction}

Parenteral nutrition (PN) is a life-saving treatment for infants and children who are unable to adequately absorb crucial nutrients due to intestinal failure [1-3]. PN's wide pediatric application significantly improves patients' nutritional statuses, which avoids the necessity for complex gastrointestinal surgery, thus increasing patient survival rates [1-3]. Nevertheless, long-term PN administration can have severe deleterious health effects, such as promoting the development of life-threatening $\mathrm{PN}$-associated liver disease (PNALD) [2]. PNALD is characterized by significant alterations to liver histology that range from hepatocyte steatosis and cholestasis to liver fibrosis, cirrhosis, or complete liver failure [2]. Importantly, PNALD is a major cause of morbidity and mortality in infants requiring long-term PN, with ten deaths and one referral for liver transplantation in the first year of life among $66 \mathrm{PN}$-associated cholestasis patients (17\%) [4]. Of the 21 infants with a maximum conjugated bilirubin $\geq 10.0 \mathrm{mg} / \mathrm{dL}$, death or liver transplantation occurred in eight (38\%) [4].

The underlying cause of PNALD onset still remains unclear. However, there is increasing evidence suggesting that the vegetable oil-based lipid emulsions used in PN treatments, which are rich in $\omega-6$ polyunsaturated fatty acids (PUFAs), likely promote PNALD development [2]. $\omega$ 6 PUFAs have been reported to increase oxidative stress, enhance inflammatory responses, and impede proper biliary secretion [5-7]. Fish oil is particularly rich in $\omega-3$ PUFAs and has anti-inflammatory properties [8]. Studies in both humans and other mammals have revealed that fish oil and fish oil-based lipid emulsions (FOLE) can prevent and alleviate PNALD [9-14]. However, the underlying reason why vegetable oil and FOLE have such dramatically different effects on PNALD remains unclear.

Lipids droplets (LD) are highly dynamic organelles that are critical for lipid storage in nearly all cell types [15]. 
Both excessive and insufficient LD concentrations are associated with a variety of human diseases. Under stress conditions, such as excessive nutrient intake and drug-induced liver injury, hepatocyte LD accumulation is apparent. This LD accumulation is an early manifestation of liver injury and ultimately causes liver steatosis [16, 17].

The endoplasmic reticulum (ER) is a cellular organelle that functions in protein synthesis, folding, modification, assembly, transport, and storage. A lapse in ER function often results in the accumulation of misfolded proteins within the ER, ultimately causing ER stress and the activation of the unfolded protein response (UPS) $[18,19]$. The glucoseregulated proteins (GRP), GRP78 and GRP94, play important roles during UPS to maintain ER homeostasis [20].

Several previous studies suggest that LD originates in the ER [17]. ER stress is thought to play a role in several liver diseases and to be related to lipid homeostasis [18]. In addition, the UPS is also activated in several liver diseases, including obesity-associated fatty liver disease, viral hepatitis, and alcohol-induced liver injury [18]. It was also recently reported that GRP94 is involved in liver tumorigenesis [21, 22]. Our previous studies also demonstrated that GRP94 mRNA and protein levels are upregulated in newborn rabbits that were intravenously treated with soybean oil-based PN. Nevertheless, GRP94 levels remained unchanged in newborn rabbits treated with fish oil-based PN [23]. We hypothesized that soybean oil-based lipid emulsions (SOLE) and FOLE may have distinctive effects on hepatocyte LD accumulation and ER stress. In this study, we use primary rabbit newborn hepatocytes to determine the effects of SOLE and FOLE on hepatocyte homeostasis.

\section{Materials and Methods}

2.1. Isolation and Treatment of Primary Hepatocytes. The animal protocols used in these studies were approved by the Animal Ethics Committee of the Children's Hospital of Soochow University. Eight seven-day-old New Zealand white rabbits (weighing 50-120 g) were obtained from Wuxi Huishan Jiangnan Experimental Animal Centre (animal license number SCXK [Su] 2009-0005) in Jiangsu, China. All rabbits were subjected to a 12/12-hour light-dark cycle and kept in an incubator that was maintained at $26^{\circ} \mathrm{C}$ to $28^{\circ} \mathrm{C}$ with $40 \%$ to $60 \%$ humidity.

Isolation of primary hepatocytes was performed using direct liver insolation and collagenase digestion, as previously reported [24]. Briefly, rabbits were anaesthetized with $3 \%$ pentobarbital sodium $(1 \mathrm{~mL} / \mathrm{kg})$ before their livers were removed and placed in $\mathrm{PBS}$ at $4^{\circ} \mathrm{C}$. Following several washes, livers were cut into multiple pieces $\left(1 \mathrm{~mm}^{3}\right)$. These pieces were centrifuged at $800 \mathrm{rpm} / \mathrm{min}$ for $6 \mathrm{~min}$ and the resulting pellet was collected and digested with collagenase I (Sigma-Aldrich, St. Louis, MO, USA) at $37^{\circ} \mathrm{C}$ for $30 \mathrm{~min}$. The suspension was then filtered through a 100-mesh copper net. After centrifugation at $500 \mathrm{rpm} / \mathrm{min}$ for $10 \mathrm{~min}$, hepatocytes were suspended in DMEM and centrifuged again. Following three cycles, hepatocytes were maintained in DMEM (Gibco of Thermo Fisher Scientific, Waltham, MA, USA) with 10\% FBS (Sijiqing,
Hangzhou, China) containing $100 \mathrm{U} / \mathrm{mL}$ penicillin, $100 \mathrm{U} / \mathrm{mL}$ streptomycin, $30 \mathrm{~g} / \mathrm{L}$ glutamine, $5 \mu \mathrm{g} / \mathrm{mL}$ insulin, and $1 \times$ $10^{-6} \mathrm{~mol} / \mathrm{L}$ hydrocortisone at a density of $2 \times 10^{4}$ cells $/ \mathrm{mL}$. Cells were cultured in a $5 \% \mathrm{CO}_{2}$ incubator at $37^{\circ} \mathrm{C}$. Media were changed after $24 \mathrm{~h}$, and then every two days thereafter. Primary hepatocytes were identified using periodic acidSchiff staining (PAS). Cells were passaged upon reaching $80 \%$ confluence.

Primary hepatocytes were treated with varying concentrations of SOLE $(0.2 \%, 0.4 \%, 1 \%$, and $2 \%$ diluted in DMEM from $20 \%$ SOLE). The $0.4 \%$ and $1 \%$ concentrations were used for all follow-up experiments. Hepatocytes were then divided into five groups: control group (CON), $0.4 \%$ or $1 \%$ FOLE (diluted from 10\% FOLE) treatment (FO Low, FO High) and $0.4 \%$ or $1 \%$ SOLE treatment (SO Low, SO High). SOLE (20\%) and FOLE (10\%) were both purchased from Sino-Swed Pharmaceutical, China.

2.2. MTT Assay. Primary hepatocytes were seeded into 96well plates at a density of $2 \times 10^{4}$ cells per well and treated as previously described. Cells were cultured for 24, 48, 72, or $96 \mathrm{~h}$. At each indicated time, MTT was added to each individual well at a final concentration of $0.5 \mathrm{mg} / \mathrm{mL}$. Cells were then incubated at $37^{\circ} \mathrm{C}$ for an additional $4 \mathrm{~h}$. Following incubation, the supernatant was removed and the cells were lysed in $200 \mu \mathrm{L}$ DMSO (Sinopharm Chemical Reagent Co. Ltd., Shanghai, China). The absorbance of the blue formazan derivative was measured at $490 \mathrm{~nm}$ using a microplate reader (DNM-9602; Shengke, Shanghai, China). All measurements were performed in triplicate and all experiments were repeated three times.

2.3. LD Accumulation Detection Using Oil Red O Staining. Primary hepatocytes were seeded into 24-well plates with polylysine-pretreated slides at a density of $2 \times 10^{4}$ cells per well. After $24 \mathrm{~h}$, cells were treated with different lipid emulsion types and concentrations, as previously described. At $24,48,72$, and $96 \mathrm{~h}$ posttreatment, the slides were fixed in $10 \%(\mathrm{v} / \mathrm{v})$ formaldehyde for $10 \mathrm{~min}$ and then stained with red oil $\mathrm{O}$ solution $(0.5 \%$ in isopropanol, w/v) for $15 \mathrm{~min}$. Following three washes with distilled water, slides were stained with hematoxylin for $10 \mathrm{~min}$. The stained slides were imaged using a light microscope (B-Type; Qiyue, Shanghai, China).

2.4. Biochemical Tests. Culture media from the five experimental groups were collected at $0,24,48$, and $72 \mathrm{~h}$. Various biochemical parameters, including total bilirubin (TBIL), direct bilirubin (DBIL), alanine aminotransferase (ALT), aspartate aminotransferase (AST), gamma glutamyltranspeptidase $(\gamma$-GT), total protein (TP), prealbumin (PA), albumin (ALB), triglycerides (TG), and lactate dehydrogenase (LDH), were measured from each sample using an automatic biochemical analyzer (LXTM20; Beckman Coulter, Inc., Brea, CA, USA).

2.5. Histologic Analysis Using Transmission Electron Microscopy (TEM). Primary hepatocytes from all five groups 
were collected at $24 \mathrm{~h}$ posttreatment and centrifuged at $1500 \mathrm{rpm} / \mathrm{min}$ for $5 \mathrm{~min}$. Cell pellets were fixed in $2.5 \%(\mathrm{v} / \mathrm{v})$ glutaraldehyde ( $\mathrm{pH}$ 7.4-7.6) for $2 \mathrm{~h}$. Cells were then pelleted by centrifugation, washed with PBS, and then fixed with $1 \%(\mathrm{w} / \mathrm{v})$ osmium tetroxide (Sigma-Aldrich) for $2 \mathrm{~h}$ at room temperature. Following dehydration with graded series of ethanol (70-100\%), cells were embedded in propylene oxide and Epon (Sigma-Aldrich) and solidified in 100\% epoxy resin at $60^{\circ} \mathrm{C}$ for $48 \mathrm{~h}$ in preparation for generating $90 \mathrm{~nm}$ ultrathin sections. Tissue sections were stained with uranyl acetate (2\%) and lead citrate (1\%) and then imaged using TEM (Tecnai G2 F20 S-TWIN; FEI, Hillsboro, OR, USA).

2.6. Quantitative RT-PCR. Total RNA was isolated from hepatocytes from each of the five experimental groups at 24, 48, and $72 \mathrm{~h}$ posttreatment using Trizol (Invitrogen of Thermo Fisher Scientific) and then reverse-transcribed into cDNA using reverse transcriptase (M-MLV; Promega Inc., Madison, WI, USA), according to the manufacturers' protocol. cDNA samples were then amplified using RealTime PCR (LightCycler 480 II; Roche, Basel, Switzerland). Conditions for PCR amplification were set to an initial $95^{\circ} \mathrm{C}$ for $10 \mathrm{~min}$, which was then followed by 40 cycles of $95^{\circ} \mathrm{C}$ for $15 \mathrm{~s}, 55^{\circ} \mathrm{C}$ for $30 \mathrm{~s}$, and $72^{\circ} \mathrm{C}$ for $35 \mathrm{~s}$. The GPR94 primers used were forward $5^{\prime}$-GACCCTCCAGCAGCATAA- $3^{\prime}$ and reverse $5^{\prime}$-AGAAGCCGCTCAACAAAT- $3^{\prime}$. The $\beta$-actin (control) primers used were forward $5^{\prime}$-GGTCGGAGTGAACGGATTT- $3^{\prime}$ and reverse $5^{\prime}$-CTCGCTCCTGGAAGATGG-3'. Quantitative PCR was performed in triplicate, and the relative mRNA expression levels were analyzed using the $2^{-\Delta \Delta \mathrm{Ct}}$ method.

2.7. Immunohistochemistry. Primary hepatocytes were seeded into 96-well plates with polylysine-pretreated slides at a density of $2 \times 10^{4}$ cells per well. After $24 \mathrm{~h}$, cells were treated with different lipid emulsions, as described above. The slides were fixed in $95 \%$ ethanol for $20 \mathrm{~min}$ at room temperature. After washing with PBS three times, cells were incubated first in blocking buffer at $37^{\circ} \mathrm{C}$ (PBS-containing goat serum) for at least $30 \mathrm{~min}$, and then overnight at $4^{\circ} \mathrm{C}$ in binding buffer (PBS containing 3\% BSA and $0.3 \%$ Triton X-100) containing rabbit anti-GRP94 polyclonal antibody (Maixin, Fuzhou, China) at a 1:200 dilution. After three PBS washes, cells were incubated with binding buffer containing HRP-conjugated goat anti-rabbit IgG (Maixin) at a 1:100 dilution for $1 \mathrm{~h}$ at room temperature. GRP94 staining was imaged under a light microscope using DAB solution (Maixin). Slides were then stained with hematoxylin for $10 \mathrm{~min}$. Stained samples were then reexamined using the light microscope. Three visual fields were randomly selected under 400x magnification and the mean protein signal intensity was quantified using image-pro-plus software (Olympus, Inc., Tokyo, Japan).

2.8. Statistical Analyses. All data are represented as the mean \pm standard deviation and were analyzed using SPSS 17.0 software (SPSS Inc., Chicago, IL, USA). The differences between groups were analyzed using a one-way ANOVA with a post hoc Bonferroni test. A $p$ value $<0.05$ was considered as statistically significant.

\section{Results}

3.1. Analysis of Cell Viability. Primary hepatocytes were isolated and identified using PAS staining (see Supplementary Figure 1 in Supplementary Material available online at http://dx.doi.org/10.1155/2016/9717014). To determine the optimal lipid emulsion concentration to be used in these experiments, we first determined the extent of SOLE-induced cytotoxicity on hepatocytes at various time points using an MTT assay. As displayed in Supplementary Figure 2A, 0.2\% and $0.4 \%$ SOLE had no detectable cytotoxic effects at all time points, $1 \%$ SOLE showed minor cytotoxicity at only $96 \mathrm{~h}$, and $2 \%$ SOLE showed significant cytotoxicity at every time point. Therefore, $0.4 \%$ and $1 \%$ lipid emulsion concentrations were used for all follow-up experiments.

The SOLE and FOLE cytotoxicities at $0.2 \%$ and $0.4 \%$ concentrations were also examined. These data revealed that only $1 \%$ SOLE caused minor cytotoxicity at $96 \mathrm{~h}$ and FOLE had no cytotoxic effects at either of these two concentrations (Supplementary Figure 2B).

\subsection{High SOLE Concentration Affects Hepatocyte Func-} tion. When PANLD occurs, several biochemical parameters including TBIL, DBIL, AST, and $\gamma$-GT are consistently increased. At the same time, liver damage destroys hepatocytes and alters their permeability, causing them to release more LDH and ALT. Liver damage also results in lower ALB synthesis. We therefore analyzed a variety of biochemical parameters to determine the effect of each treatment on hepatocyte function. As shown in Figure 1, the levels of TBIL, DBIL, ALT, AST, $\gamma$-GT, and LDH were all significantly higher in the SO High group when compared with all other groups at all time points (all $p<0.05$ ). Furthermore, ALB levels were significantly lower in the SO High group compared with the other groups at each of the time points (all $p<0.05$ ). No significant differences in TG, PA, or TP were observed among the groups at any of the time points. Additionally, no significant differences were observed in any of the parameters among the FO High, FO Low, SO Low, and CON groups at any of the time points.

3.3. Fish Oil and Soybean Oil Have Different Effects on Hepatocyte Function. We analyzed the effects of FOLE and SOLE on hepatocyte $\mathrm{LD}$ accumulation using oil red $\mathrm{O}$ staining. As shown in Figure 2, hepatocytes in the CON group showed normal morphologies, with sharpened edges and limited red labeling at all time points. FO Low group hepatocytes showed normal morphologies at every time point and there were few red LD that were only visible at $96 \mathrm{~h}$. The FO High group hepatocytes also exhibited normal morphologies at 24 and $48 \mathrm{~h}$ and showed minimal cell rounding at $72 \mathrm{~h}$ that compromised intercellular connections. In addition, red LD appeared in this group at $24 \mathrm{~h}$ and gradually increased throughout the subsequent time points. Nevertheless, although the SO Low group cells showed normal morphologies at all time points 
TBIL
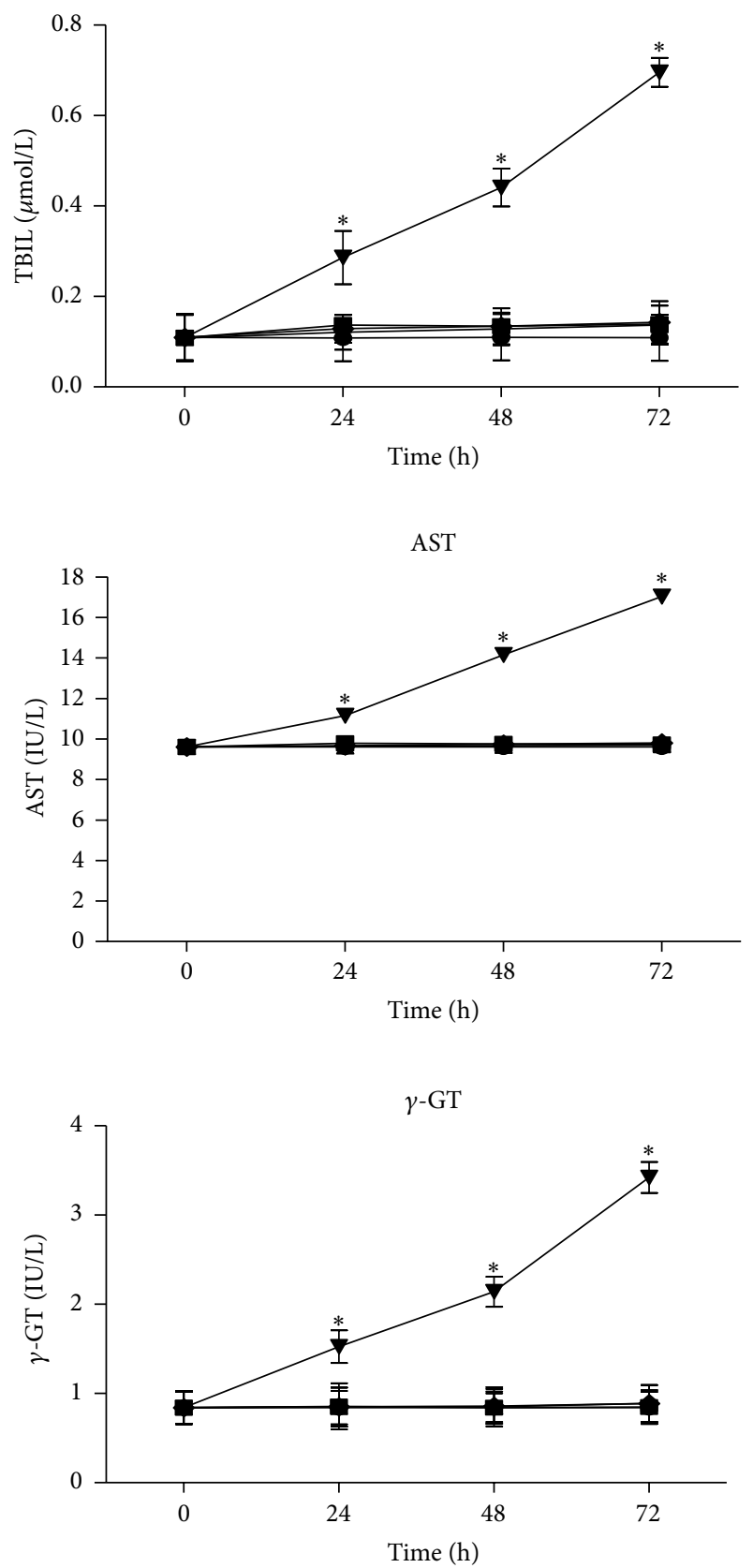

DBIL

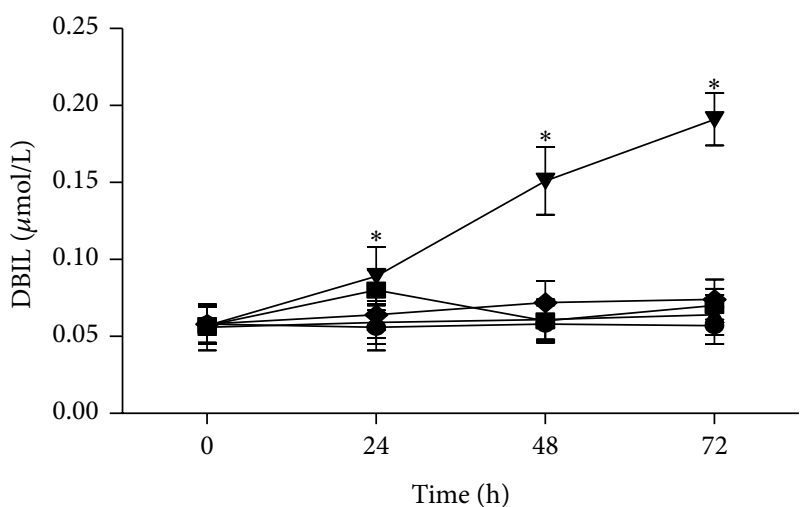

DBIL

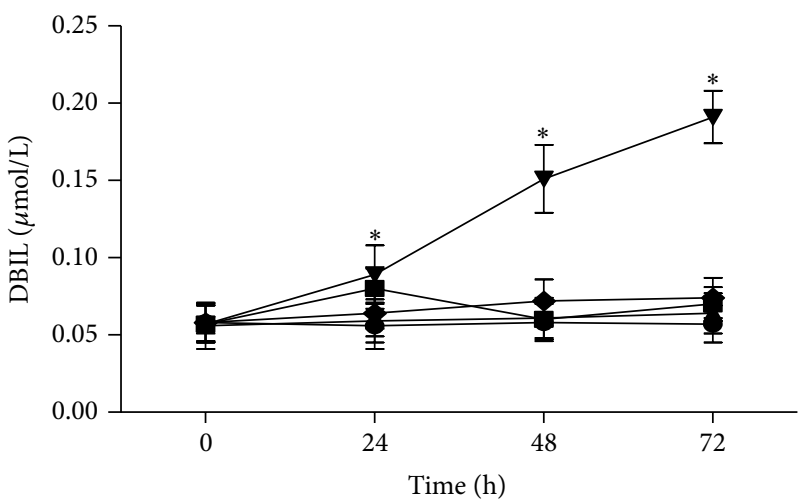

ALT
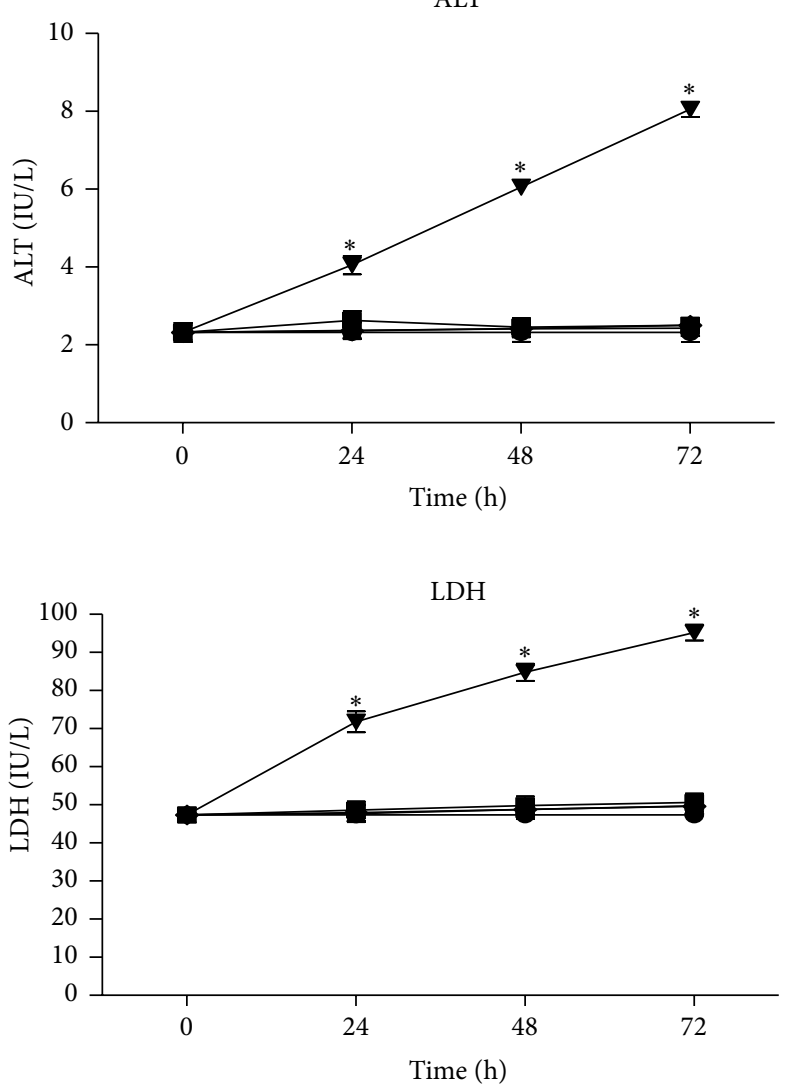

TG

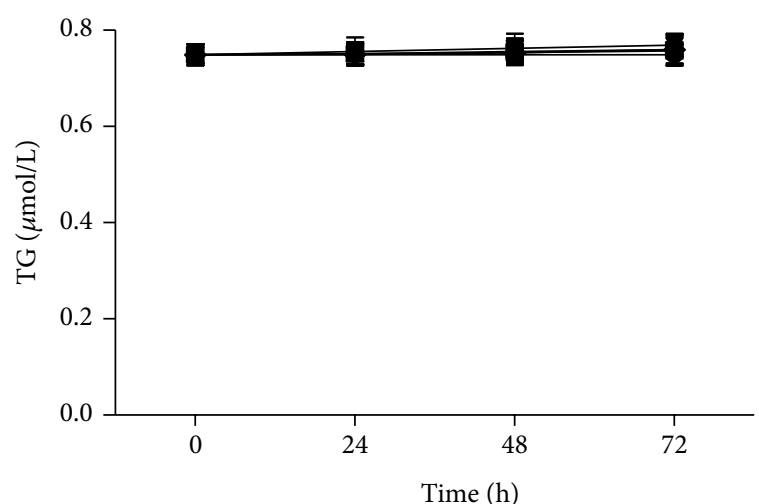

Figure 1: Continued. 

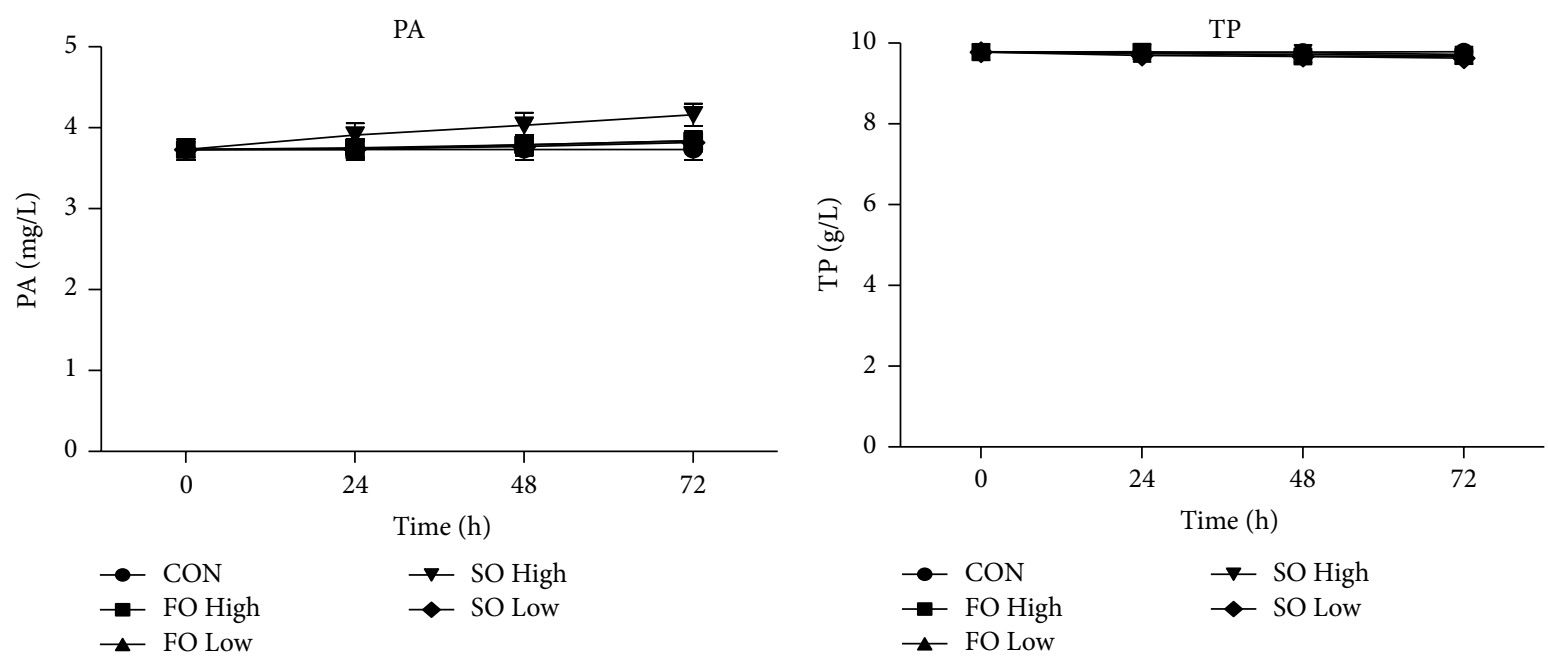

Figure 1: High concentrations of soybean oil affect hepatocyte function. Primary hepatocytes were treated with $0.4 \%$ or $1 \%$ soybean or fish oil-based lipid emulsions and several biochemical parameters were analyzed at the indicated time points to determine hepatic function; ${ }^{*} p<0.05$ versus CON. TBIL, total bilirubin; DBIL, direct bilirubin; ALT, alanine aminotransferase; AST, aspartate aminotransferase; $\gamma$-GT, gamma-glutamyltranspeptidase; TP, total protein; PA, prealbumin; ALB, albumin; TG, triglycerides; LDH, lactate dehydrogenase.
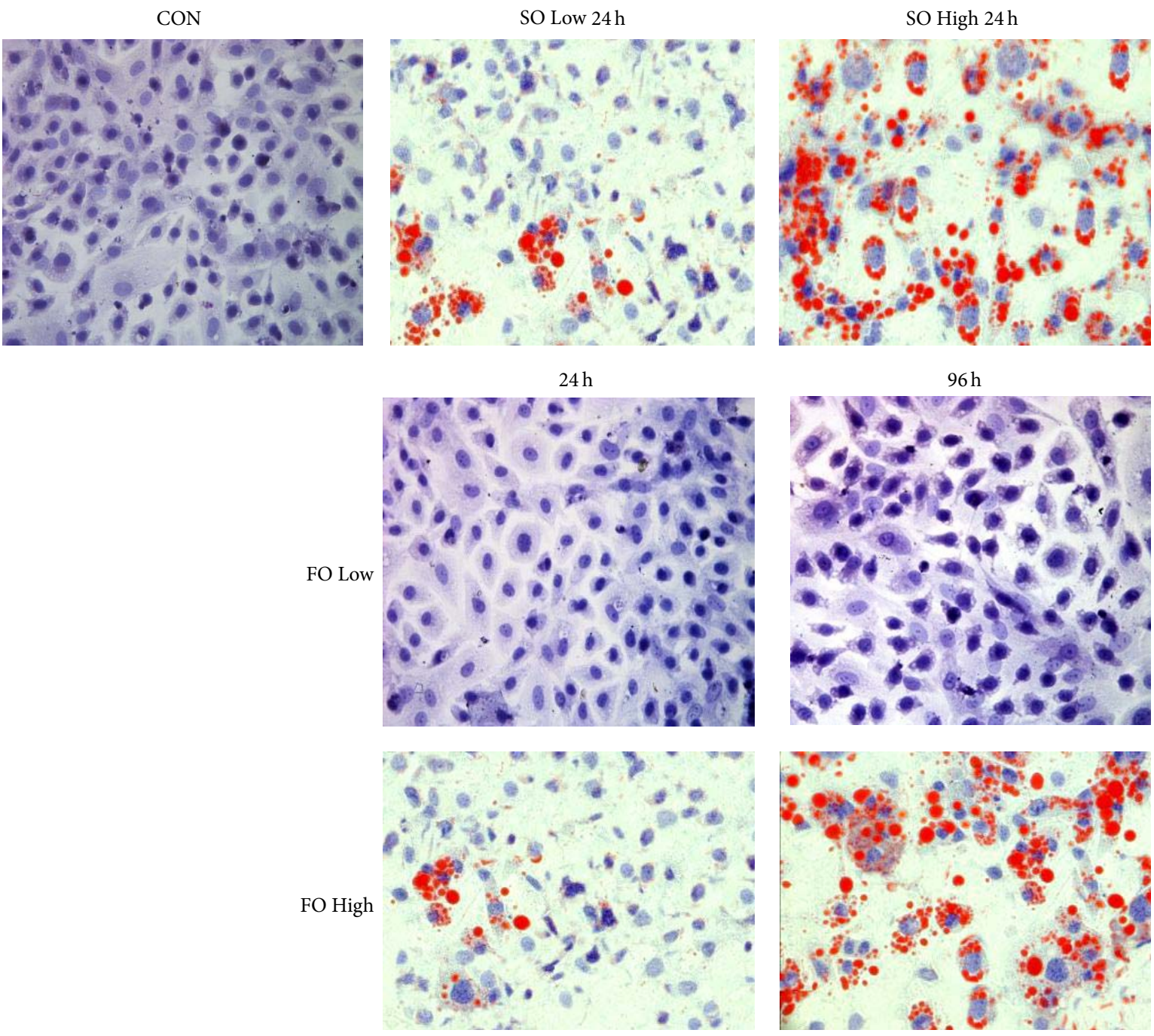

FIGURE 2: Soybean oils cause greater hepatocyte injury and LD accumulation than fish oil. Primary hepatocytes were treated with $0.4 \%$ or $1 \%$ soybean or fish oil-based lipid emulsions and cellular LD accumulation was detected using oil red O staining. LD, lipid droplets. 


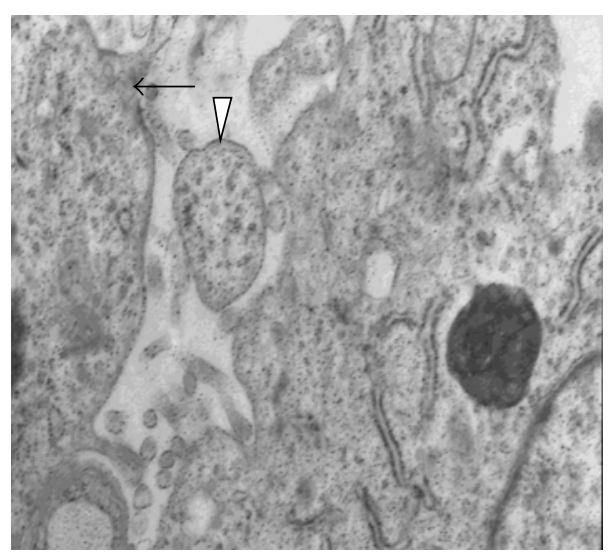

(a)

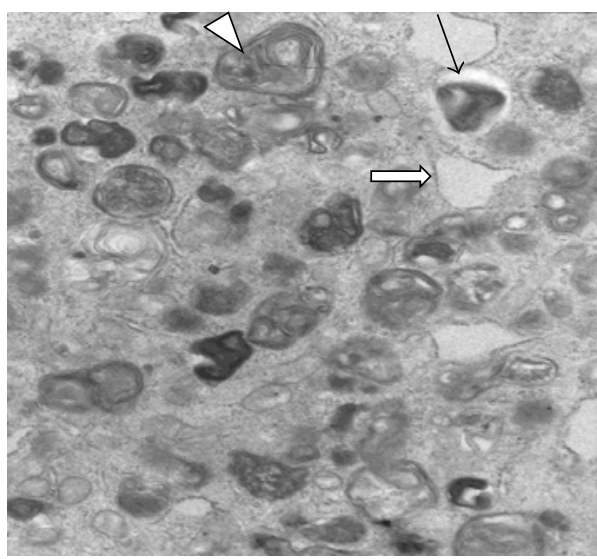

(c)

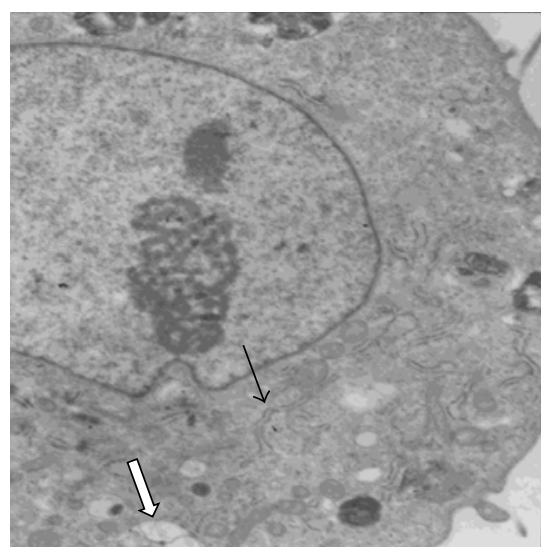

(b)

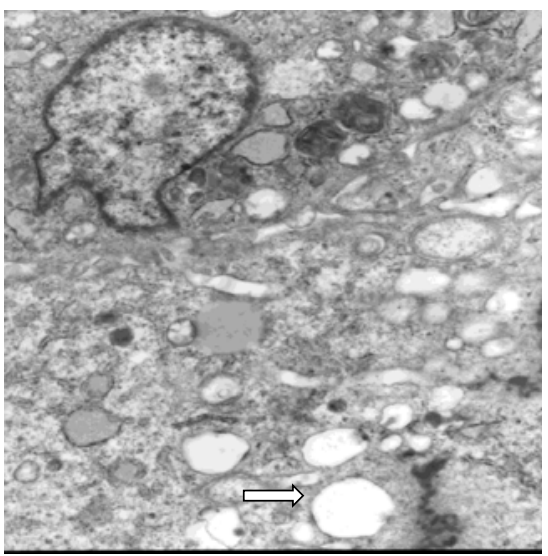

(d)

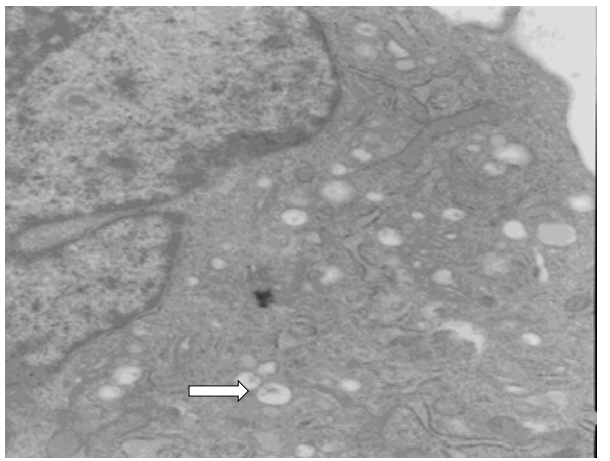

(e)

FIGURE 3: Soybean oils but not fish oils cause ER and mitochondrial damage. Primary hepatocytes were treated with $0.4 \%$ or $1 \%$ soybean (SO) or fish oil- (FO-) based lipid emulsions and the changes to both ER and mitochondrial morphologies were examined using TEM $(\times 10000)$. (a) CON group, (b) FO High group, (c) FO Low group, (d) SO High group, and (e) SO Low group. White filled triangle indicates mitochondria; black filled arrow indicates ER; white filled arrow indicates lipid droplets. ER, endoplasmic reticulum; TEM, transmission electron microscopy.

besides $96 \mathrm{~h}$, including a few rounded cells, red LD appeared at $24 \mathrm{~h}$ and increased gradually in the proceeding time points. Moreover, cells in the SO High group became rounded at $24 \mathrm{~h}$ with many red LD also apparent, and these cells gradually exhibited blurred edges with LD fused circles around the inner membrane.
3.4. Soybean Oil Causes ER and Mitochondrial Damage. We then used TEM to observe any alterations to hepatocyte ER morphology that were caused by the different lipid emulsions. Cells in the CON group demonstrated normal volumes, ER, and mitochondrial morphologies and lacked LDs (Figure 3(a)). Furthermore, cells in the FO High group 
showed slightly larger volumes, minor ER expansion, some mitochondrial swelling, the absence of mitochondrial crests, and few LDs (Figure 3(b)). Cells in the FO Low group exhibited normal cell volumes, normal ER and mitochondrial morphologies, and no LDs (Figure 3(c)). However, the majority of the cells in the SO High group showed a significantly larger volume, dramatic ER expansion, including a few fractured vacuole-containing $\mathrm{ER}$, some mitochondrial swelling, the absence of mitochondria crests, and many LDs (Figure 3(d)). Similar to those in the FO High group, cells in the SO Low group also showed a slightly larger volume, minor ER expansion, minor mitochondrial swelling, the absence of mitochondria crests, and some LD (Figure 3(e)).

3.5. Soybean Oil Significantly Upregulates GRP94 mRNA and Protein Expression. We also determined the amount of GRP94 expression, which reflects the UPS during ER stress, in the different lipid emulsions. As shown in Figure 4, both the mRNA and protein levels of GRP94 in all except the SO high group showed no differences when compared with the CON group at any of the time points. The SO high group, however, showed significantly higher GRP94 expression levels at every time point (all $p<0.05$ ).

\section{Discussion}

In this study, we reveal the differential effects of soybean and fish oil on hepatocyte function and structural integrity. The changes in several biochemical parameters that reflect hepatic function, which include LD accumulation, ER and mitochondrial structures, and ER stress, were compared between primary newborn rabbit hepatocytes that were treated with two different oil-based lipid emulsions. Our results reveal that SOLE caused significant damage to both the ER and mitochondria, ultimately resulting in LD accumulation, ER stress, and eventual hepatocyte malfunction. Conversely, FOLE caused only minor ER and mitochondrial damage at higher doses, which did not affect hepatic function. We therefore suggest that FOLE might serve a preventative and/or protective function against PNALD.

The lipid source in parenteral lipid emulsions is thought to play an essential role in the development of PNALD [9]. Currently, lipid emulsions are primarily derived from either soybean oils alone or a mixture of soybean and safflower oil. These vegetable oil-based lipid emulsions are rich in both phytosterols as well as $\omega-6$ PUFAs. Phytosterols can accumulate in patient serum or in either cellular membranes or plasma lipoproteins, which are all associated with PNALD $[25,26]$. Arachidonic acid is an $\omega-6$ PUFA and is the precursor of the two important inflammatory mediators, namely, prostaglandins and leukotrienes. Therefore, $\omega-6$ PUFAs may either initiate or exacerbate inflammation, as well as providing immunosuppressive effects, which could potentially contribute to PNALD development and progression [27, 28]. Contrarily, $\omega-3$ PUFAs, which are abundant in fish oils, suppress inflammation by decreasing the production of inflammatory cytokines, eicosanoids, and reactive oxygen species [9, 29]. Our results also reveal that soybean oils cause significant ER and mitochondrial damage, ultimately resulting in LD accumulation within hepatocytes. Fish oil, on the other hand, showed no such effects. Hepatocyte injury can thus be alleviated in PN patients by using fish oils instead of vegetable oils in order to decrease the likelihood of causing PNALD.

Clinical trials suggest that the PNALD onset is positively correlated with both the timespan and dose of lipid emulsions applied $[9,30]$. Our data are consistent with this correlation, revealing that increasing doses of soybean oil for longer timespans cause increasingly deleterious effects to hepatocyte function. High doses of fish oils over a long timespan also cause minor hepatocyte damage. However, although LD accumulation was consistently observed in SOLE-treated hepatocytes, there were no significant TG changes in the supernatant. The reason for this phenomenon could be attributable to the fact that the culture media used are different from the in vivo environment of hepatocytes. Whereas hormones in the body stimulate the release of TG, our culture media may not efficiently elicit this response.

Our results also show that while soybean oils induce ER stress and upregulate GRP94 expression in hepatocytes, fish oils have no such effect. ER stress is an adaptive cellular response to multiple stimuli and is linked to several diseases [31]. Persistent ER stress ultimately promotes apoptosis [19]. ER stress and the resulting apoptosis contribute to the development of many liver diseases, including alcoholic liver disease, nonalcoholic fatty liver disease, viral hepatitis, acute liver failure, and hepatocellular carcinoma [18, 3234]. Previous work revealed that ER disorder and hepatic microvesicular steatosis were caused by the genetic ablation of ER stress-sensing pathways in mice [35]. In addition, the rescue of ER protein-processing capacity was shown to prevent the suppression of a subset of metabolic transcription factors that regulate lipid homeostasis. As hepatic steatosis also occurs during PNALD, it is possible that ER stress is also involved in this process.

GRP94 and GRP78 are two of the major proteins that are involved in UPS [36]. The upregulation of both GRP94 and GRP78 is a hallmark of ER stress. Our previous study demonstrated that GRP94 is upregulated in rabbits that receive $\mathrm{PN}$ containing soybean oil-based lipid emulsions. In this study, we further confirm that soybean oils directly affect hepatic function by upregulating GRP94. Recently, GRP94 was reported to be involved in hepatocellular carcinogenesis [37]. This suggests that soybean oil-driven GRP94 upregulation in hepatocytes may play an important role in PNALD development.

In conclusion, we reveal that soybean oils and not fish oils damage the ER and mitochondria, resulting in LD accumulation and ER stress. This ultimately induces hepatocyte injury, which likely explains the differential impacts of soybean and fish oils on PNALD development and progression.

\section{Conflict of Interests}

The authors declare that they have no conflict of interests concerning this paper. 


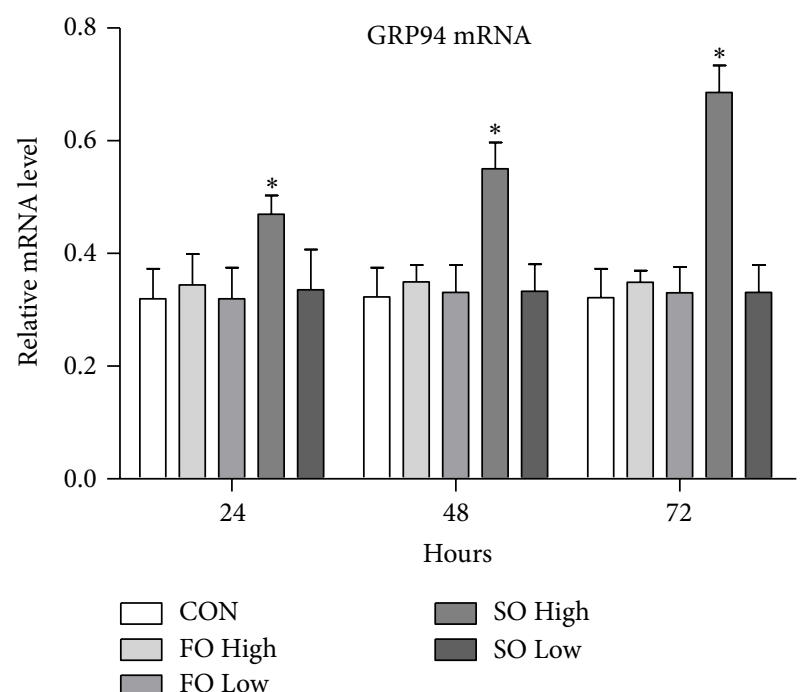

(a)

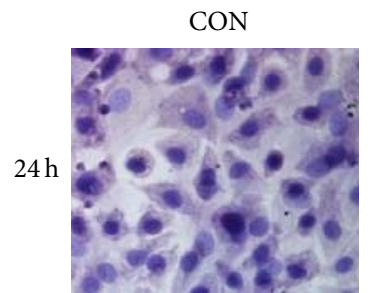

$48 \mathrm{~h}$
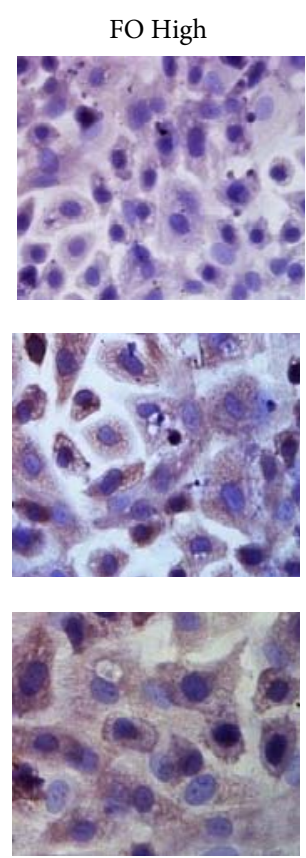

$$
72 \mathrm{~h}
$$

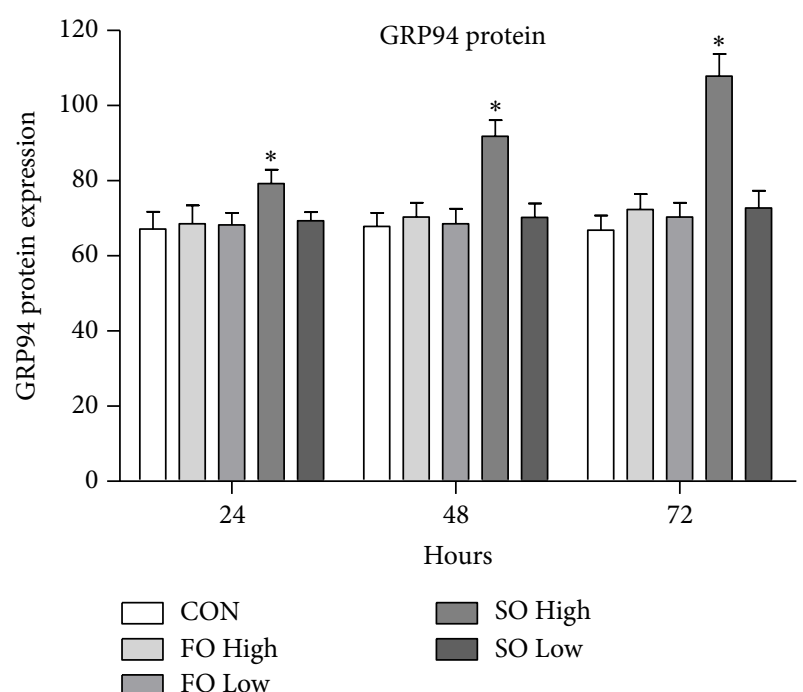

(c)
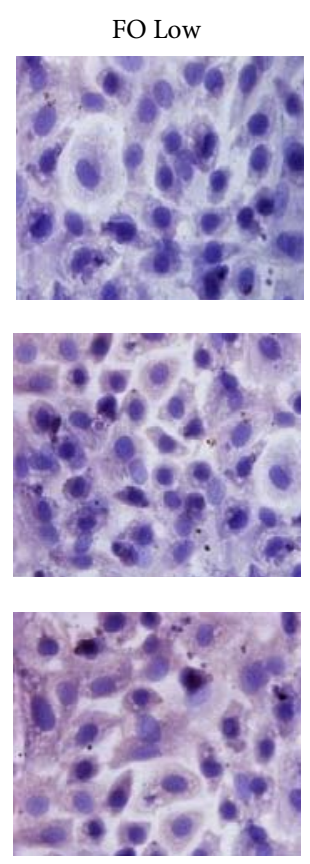

(b)
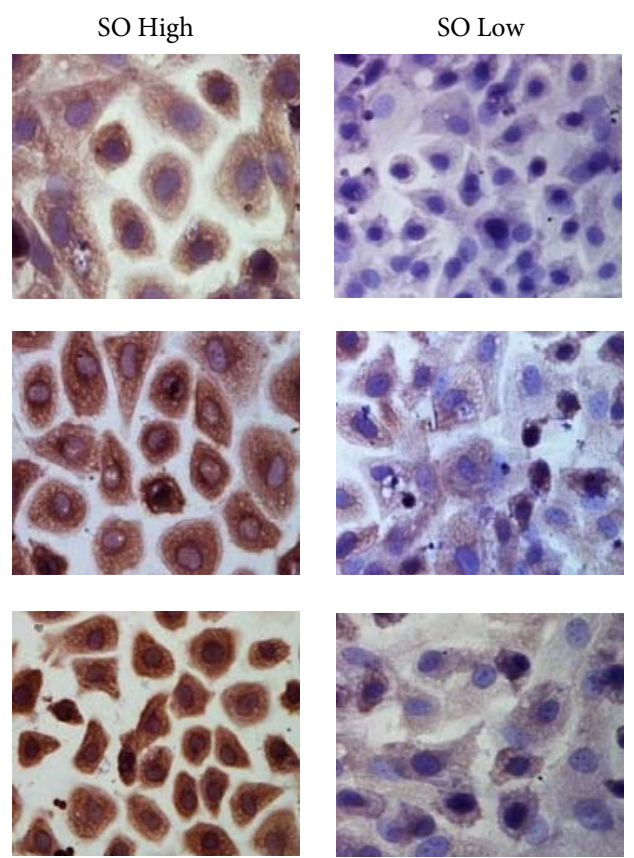

FIgURE 4: Soybean oils but not fish oils upregulate GRP94 mRNA and protein levels. Primary hepatocytes were treated with $0.4 \%$ or $1 \%$ soybean or fish oil-based lipid emulsions. The GPR94 mRNA expression (a) was detected using qRT-PCR and GRP94 protein expression (b, c) was detected by immunohistochemistry at the indicated time points; ${ }^{*} p<0.05$ versus CON. GRP, glucose-regulated protein.

\section{Authors' Contribution}

Xueping Zhu participated in study design and data analysis, interpretation of data, and writing of the paper. Zhihui Xiao participated in the data analysis and interpretation of data. Yumin Xu carried out the cell culture, immunoassays, and RT-PCR. Xingli Zhao and Ping Cheng participated in the sequence alignment. Ningxun Cui, Mingling Cui, and Jie Li participated in the sequence alignment and assisted with the RT-PCR. All authors read and approved the final paper. Xiaoli Zhu participated in study design and interpretation of data. Xueping Zhu and Zhihui Xiao contributed equally to this work.

\section{Acknowledgments}

This research was supported by grants from Suzhou Science and Technology Development Project (SYS201136 and 
SYS201440) and Jiangsu Province Health Department Surface Scientific Research Project (H201316), Natural Science Foundation Project of Jiangsu Province (no. BK20141183), 135 Project of Department of Health of Jiangsu Province (no. RC2007076), and Research Project of the Suzhou Key Laboratory of Children's Developmental Brain Injury Prevention and Care (no. SZS201108). The authors gratefully acknowledge all members of the laboratory for sharing reagents and advice. The authors also thank the pathologist for reviewing the histology slides.

\section{References}

[1] E. Cowan, P. Nandivada, and M. Puder, "Fish oil-based lipid emulsion in the treatment of parenteral nutrition-associated liver disease," Current Opinion in Pediatrics, vol. 25, no. 2, pp. 193-200, 2013.

[2] E. M. Fallon, H. D. Le, and M. Puder, "Prevention of parenteral nutrition-associated liver disease: role of omega-3 fish oil," Current Opinion in Organ Transplantation, vol. 15, no. 3, pp. 334-340, 2010.

[3] M. P. Cober and D. H. Teitelbaum, "Prevention of parenteral nutrition-associated liver disease: lipid minimization," Current Opinion in Organ Transplantation, vol. 15, no. 3, pp. 330-333, 2010.

[4] T. C. Willis, B. A. Carter, S. P. Rogers, K. M. Hawthorne, P. D. Hicks, and S. A. Abrams, "High rates of mortality and morbidity occur in infants with parenteral nutrition-associated cholestasis," Journal of Parenteral and Enteral Nutrition, vol. 34, no. 1, pp. 32-37, 2010.

[5] O. Goulet, F. Joly, O. Corriol, and V. Colomb-Jung, "Some new insights in intestinal failure-associated liver disease," Current Opinion in Organ Transplantation, vol. 14, no. 3, pp. 256-261, 2009.

[6] G. Szabo, P. Mandrekar, and A. Dolganiuc, "Innate immune response and hepatic inflammation," Seminars in Liver Disease, vol. 27, no. 4, pp. 339-350, 2007.

[7] L. Schaeffer, H. Gohlke, M. Müller et al., "Common genetic variants of the FADS1 FADS2 gene cluster and their reconstructed haplotypes are associated with the fatty acid composition in phospholipids," Human Molecular Genetics, vol. 15, no. 11, pp. 1745-1756, 2006.

[8] P. C. Calder, S. Krauss-Etschmann, E. C. de Jong et al., "Early nutrition and immunity-progress and perspectives," British Journal of Nutrition, vol. 96, no. 4, pp. 774-790, 2006.

[9] V. E. de Meijer, K. M. Gura, J. A. Meisel, H. D. Le, and M. Puder, "Parenteral fish oil monotherapy in the management of patients with parenteral nutrition-associated liver disease," Archives of Surgery, vol. 145, no. 6, pp. 547-551, 2010.

[10] M. Puder, C. Valim, J. A. Meisel et al., "Parenteral fish oil improves outcomes in patients with parenteral nutrition-associated liver injury," Annals of Surgery, vol. 250, no. 3, pp. 395402, 2009.

[11] H. D. Le, V. E. de Meijer, D. Zurakowski, J. A. Meisel, K. M. Gura, and M. Puder, "Parenteral fish oil as monotherapy improves lipid profiles in children with parenteral nutrition-associated liver disease," Journal of Parenteral and Enteral Nutrition, vol. 34, no. 5, pp. 477-484, 2010.

[12] M. H. Premkumar, B. A. Carter, K. M. Hawthorne, K. King, and S. A. Abrams, "High rates of resolution of cholestasis in parenteral nutrition-associated liver disease with fish oil-based lipid emulsion monotherapy," The Journal of Pediatrics, vol. 162, no. 4, pp. 793.e1-798.e1, 2013.

[13] M. H. Premkumar, B. A. Carter, K. M. Hawthorne, K. King, and S. A. Abrams, "Fish oil-based lipid emulsions in the treatment of parenteral nutrition-associated liver disease: an ongoing positive experience," Advances in Nutrition, vol. 5, no. 1, pp. 6570, 2014.

[14] W.-J. Chen and S.-L. Yeh, "Effects of fish oil in parenteral nutrition," Nutrition, vol. 19, no. 3, pp. 275-279, 2003.

[15] N. Krahmer, R. V. Farese Jr., and T. C. Walther, "Balancing the fat: lipid droplets and human disease," The EMBO Molecular Medicine, vol. 5, no. 7, pp. 905-915, 2013.

[16] D. Q.-H. Wang, P. Portincasa, and B. A. Neuschwander-Tetri, "Steatosis in the Liver," Comprehensive Physiology, vol. 3, no. 4, pp. 1493-1532, 2013.

[17] N. Sahini and J. Borlak, "Recent insights into the molecular pathophysiology of lipid droplet formation in hepatocytes," Progress in Lipid Research, vol. 54, no. 1, pp. 86-112, 2014.

[18] H. Malhi and R. J. Kaufman, "Endoplasmic reticulum stress in liver disease," Journal of Hepatology, vol. 54, no. 4, pp. 795-809, 2011.

[19] I. Tabas and D. Ron, "Integrating the mechanisms of apoptosis induced by endoplasmic reticulum stress," Nature Cell Biology, vol. 13, no. 3, pp. 184-190, 2011.

[20] G. Zhu and A. S. Lee, "Role of the unfolded protein response, GRP78 and GRP94 in organ homeostasis," Journal of Cellular Physiology, vol. 230, no. 7, pp. 1413-1420, 2014.

[21] W. Chen, D. Ha, G. Kanel, and A. S. Lee, "Targeted deletion of ER chaperone GRP94 in the liver results in injury, repopulation of GRP94-positive hepatocytes, and spontaneous hepatocellular carcinoma development in aged mice," Neoplasia, vol. 16, no. 8, pp. 617-626, 2014.

[22] W.-T. Chen, C.-C. Tseng, K. Pfaffenbach et al., "Liver-specific knockout of GRP94 in mice disrupts cell adhesion, activates liver progenitor cells, and accelerates liver tumorigenesis," Hepatology, vol. 59, no. 3, pp. 947-957, 2014.

[23] X. Zhu, Z. Xiao, X. Chen et al., "Parenteral nutrition-associated liver injury and increased GRP94 expression prevented by $\omega$ 3 fish oil-based lipid emulsion supplementation," Journal of Pediatric Gastroenterology and Nutrition, vol. 59, no. 6, pp. 708713, 2014

[24] C. Eeckhoutte, A. Giuliano Albo, M. Carletti et al., "Timedependent variations of drug-metabolising enzyme activities (DMEs) in primary cultures of rabbit hepatocytes," Toxicology in Vitro, vol. 16, no. 4, pp. 375-382, 2002.

[25] P. T. Clayton, A. Bowron, K. A. Mills, A. Massoud, M. Casteels, and P. J. Milla, "Phytosterolemia in children with parenteral nutrition-associated cholestatic liver disease," Gastroenterology, vol. 105, no. 6, pp. 1806-1813, 1993.

[26] P. T. Clayton, P. Whitfield, and K. Iyer, “The role of phytosterols in the pathogenesis of liver complications of pediatric parenteral nutrition," Nutrition, vol. 14, no. 1, pp. 158-164, 1998.

[27] B. S. Dunbar, R. V. Bosire, and R. J. Deckelbaum, "Omega 3 and omega 6 fatty acids in human and animal health: an African perspective," Molecular and Cellular Endocrinology, vol. 398, no. 1-2, pp. 69-77, 2014.

[28] P. C. Calder, "Use of fish oil in parenteral nutrition: rationale and reality," Proceedings of the Nutrition Society, vol. 65, no. 3, pp. 264-277, 2006.

[29] P. C. Calder, "n-3 polyunsaturated fatty acids, inflammation, and inflammatory diseases," American Journal of Clinical Nutrition, vol. 83, no. 6, supplement, pp. 1505S-1519S, 2006. 
[30] V. E. de Meijer, K. M. Gura, H. D. Le, J. A. Meisel, and M. Puder, "Fish oil-based lipid emulsions prevent and reverse parenteral nutrition-associated liver disease: the Boston experience," Journal of Parenteral and Enteral Nutrition, vol. 33, no. 5, pp. 541-547, 2009.

[31] N. Dicks, K. Gutierrez, M. Michalak, V. Bordignon, and L. B. Agellon, "Endoplasmic reticulum stress, genome damage, and cancer," Frontiers in Oncology, vol. 5, article 11, 2015.

[32] T. Asselah, I. Bièche, A. Mansouri et al., "In vivo hepatic endoplasmic reticulum stress in patients with chronic hepatitis C," Journal of Pathology, vol. 221, no. 3, pp. 264-274, 2010.

[33] C. Ji and N. Kaplowitz, "Betaine decreases hyperhomocysteinemia, endoplasmic reticulum stress, and liver injury in alcoholfed mice," Gastroenterology, vol. 124, no. 5, pp. 1488-1499, 2003.

[34] Y. Wei, D. Wang, F. Topczewski, and M. J. Pagliassotti, "Saturated fatty acids induce endoplasmic reticulum stress and apoptosis independently of ceramide in liver cells," American Journal of Physiology -Endocrinology and Metabolism, vol. 291, no. 2, pp. E275-E281, 2006.

[35] D. T. Rutkowski, J. Wu, S.-H. Back et al., "UPR pathways combine to prevent hepatic steatosis caused by ER stressmediated suppression of transcriptional master regulators," Developmental Cell, vol. 15, no. 6, pp. 829-840, 2008.

[36] Y. Ma and L. M. Hendershot, "ER chaperone functions during normal and stress conditions," Journal of Chemical Neuroanatomy, vol. 28, no. 1-2, pp. 51-65, 2004.

[37] S. Rachidi, S. Sun, B. X. Wu et al., "Endoplasmic reticulum heat shock protein gp96 maintains liver homeostasis and promotes hepatocellular carcinogenesis," Journal of Hepatology, vol. 62, no. 4, pp. 879-888, 2015. 


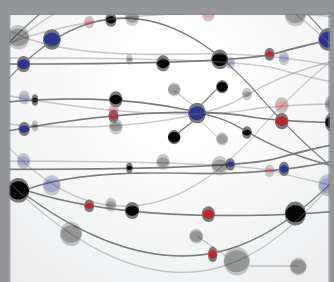

The Scientific World Journal
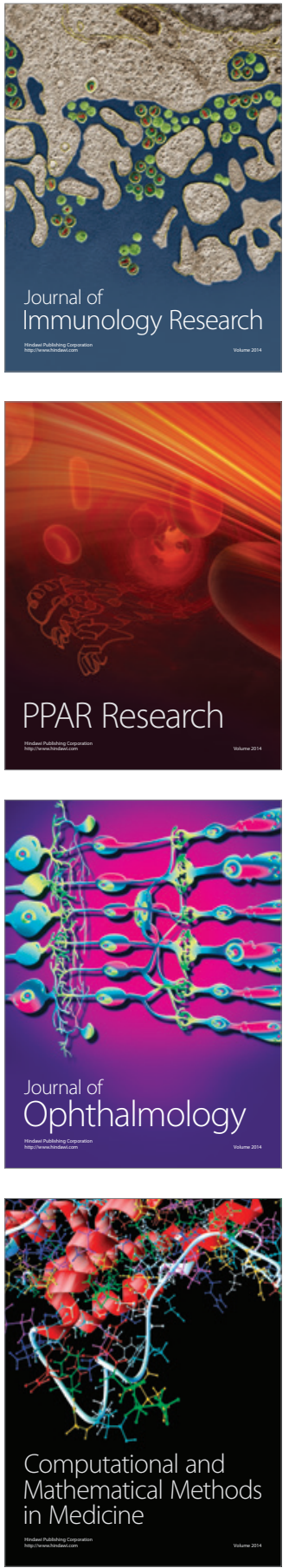

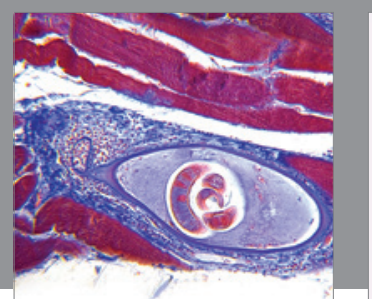

Gastroenterology Research and Practice

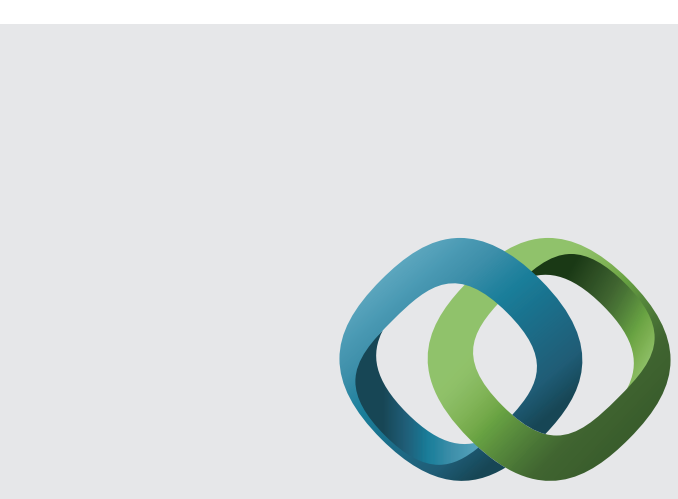

\section{Hindawi}

Submit your manuscripts at

http://www.hindawi.com
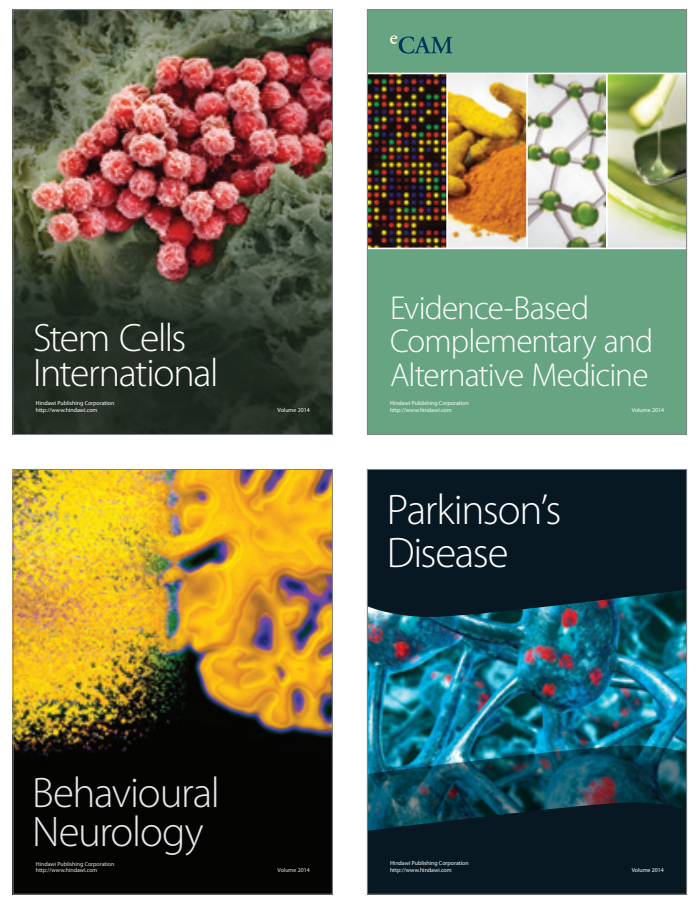
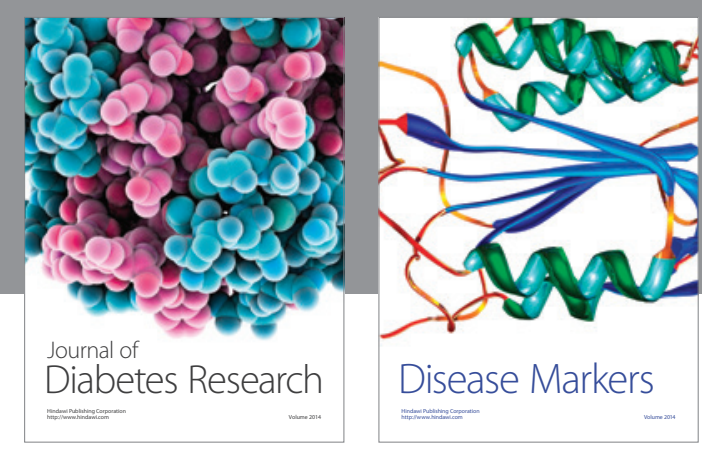

Disease Markers
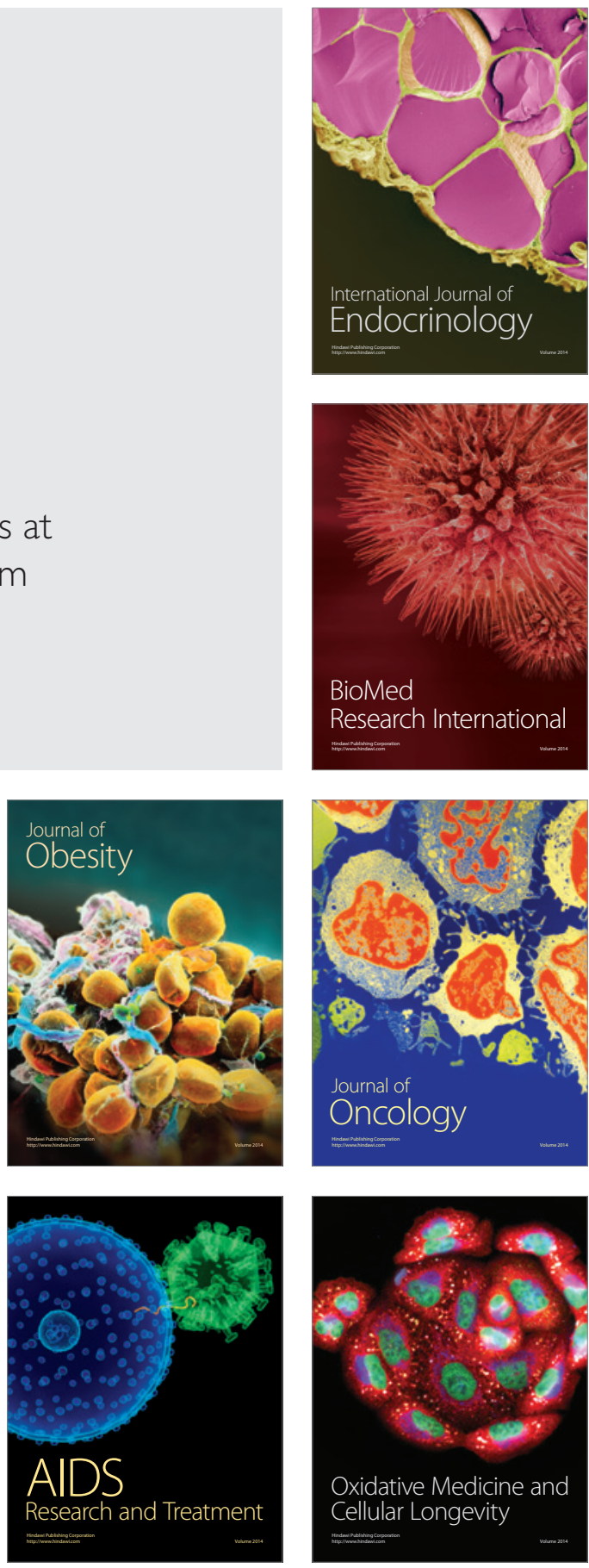